\title{
Deuterium Retention in Tungsten Used in ASDEX Upgrade: Comparison of Tokamak and Laboratory Studies
}

\author{
K. Sugiyama*, M. Mayer, A. Herrmann, K. Krieger, V. Rohde, M. Balden, S. Lindig, \\ R. Neu, H.W. Müller and the ASDEX Upgrade team \\ Max-Planck-Institut für Plasmaphysik, EURATOM Association, Boltzmannstr.2, 85748 Garching, \\ Germany
}

\begin{abstract}
Deuterium (D) retention in tungsten (W) materials, i.e. 2 types of $\mathrm{W}$ coatings and solid bulk W, used as outer divertor target in ASDEX Upgrade (AUG) was investigated and compared with laboratory experiments. The $\mathrm{D}$ concentration in the $\mathrm{W}$ coatings was larger than in solid bulk $\mathrm{W}$ because $\mathrm{W}$ coatings generally have higher inherent defect density, which is consistent with laboratory data. No clear blistering was found on the solid bulk PC-W, which could be due to its technically-machined surface. The amount of D retained in the solid bulk W tile of AUG showed a nearly square-root dependence from the incident fluence, suggesting that a diffusion-limited trapping process plays a dominant role for the D accumulation. The overall retention behaviour in AUG $\mathrm{W}$ materials was reasonably within the range of the predictions based on laboratory studies.
\end{abstract}

PACS: $52.40 . H f$ (Plasma-wall interactions; boundary layer effects)

$28.52 \mathrm{Fa}$ (Fusion Reactor Materials)

*Corresponding author

Kazuyoshi Sugiyama

Max-Planck-Institut für Plasmaphysik, EURATOM Association

Boltzmannstrasse 2, D-85748 Garching bei München, Germany

TEL: +49-(0)89-3299-1492

FAX: +49-(0)89-3299-1212

E-mail: kazuyoshi.sugiyama@ipp.mpg.de 


\section{Introduction}

Tungsten (W) is considered to be a candidate material for the plasma-facing components (PFCs) in future fusion reactors [1], and chosen as the divertor armour in ITER [2]. One advantage for use of W is a very low solubility for hydrogen, which can lead to less tritium retention in PFCs. Substantial laboratory studies regarding hydrogen retention in $\mathrm{W}$ have been performed, particularly in the last decade, in order to understand the behaviour of hydrogen in $\mathrm{W}$ and to obtain reliable models for the expected tritium inventory in reactors with W-PFCs. Although the mechanism of retention in $\mathrm{W}$ is still not fully clarified yet, those efforts in laboratories have resulted in an extensive experimental database, and also allow for extrapolations of the tritium inventory in W-PFCs [3]. In order to validate and improve these extrapolations, comparison of laboratory studies with data from existing fusion devices is a crucial issue.

ASDEX Upgrade (AUG), with its full-W plasma-facing components, has provided unique possibilities not only to develop the optimum plasma operation under a full-W wall environment but also to investigate the fuel retention in $\mathrm{W}$ used in a realistic fusion device [4]. It was found that, for instance, in-vessel fuel retention was reduced by a factor of 5-10 by replacing the plasma-facing material from full-carbon to full-W [5-7]. In AUG, several different types of W coatings (on fine grain graphite) have been utilized for the wall components. In addition, some solid bulk W were also installed as probes or as test tiles in recent campaigns. Some of those $\mathrm{W}$ tiles/probes were retrieved after each campaign, and analysed ex-situ by various methods in order to obtain the data about longterm retention of deuterium (D) in W materials under realistic fusion condition.

In this contribution we report those AUG studies on fuel retention, i.e. characteristics of D retention in AUG W materials, performed during the last few years, and compare these results to those of laboratory experiments.

\section{Experimental details}

\subsection{Analyzed tiles and plasma operation history}

The series of analyses has been performed mostly for tiles used as the outer divertor target where W will be employed in ITER, and where it receives the highest particle and heat loads among the PFCs in a tokamak. In the campaigns 2007 and 2008, a $200 \mu \mathrm{m}$ thick W coating deposited by vacuum plasma spraying (VPS) was used for outer divertor armour, and it has been exchanged before the 2009 campaign to a $10 \mu \mathrm{m}$ thick $\mathrm{W}$ coating prepared by combined magnetron sputtering and ion implantation (CMSII) deposition method [8]. The outer divertor target will be fully replaced by solid bulk polycrystalline (PC) W in the next divertor configuration (Div-III) from the campaign 2014 [9]. It is expected that the solid bulk PC-W tile can overcome potential problems of $\mathrm{W}$ coatings, such as limited lifetime due to sputtering and accidental delamination by excessive heat loads, and thereby extend the operational parameter space. Prior to the complete replacement, 2 solid bulk PC-W tiles 
were installed in the 2010/11 campaign to assess their feasibility. These tiles were also analyzed as the first solid bulk W used as an armour tile in AUG. In this report, results obtained from VPS-W coating (2007), CMSII-W coating (2009) and solid bulk PC-W (2010/11) tiles will be discussed.

The campaigns 2007, 2009 and 2010/11 consisted of 2620, 5180 and 5694 sec. of discharges, respectively, with typically lower single-null divertor configuration. The outer divertor strike point was usually located around $\mathrm{s}=1.050 \sim 1.100 \mathrm{~m}$ as shown in figure 1 . Figure 1 also shows the fluence distributions on the target tile measured by the divertor Langmuir probes, which were typically in ranges of $10^{25}-10^{26} \mathrm{~m}^{-2}$. One can calculate the ion flux to the target surface from the fluence and discharge time, and the average flux was in the order of $10^{22} \mathrm{~m}^{-2} \mathrm{~s}^{-1}$. The divertor surface temperature is routinely monitored by IR thermography. The surface temperature depends on the operation scenario, e.g. temperature excursions above $1000 \mathrm{~K}$ (up to $1200 \mathrm{~K}$ in 2010/11 campaign) during ELMs with very short time intervals of a few $\mathrm{ms}$ are occasionally observed. However, the mean surface temperature during a discharge is $400-500 \mathrm{~K}$ in most cases.

\subsection{Post mortem analyses of $A U G W$ tiles}

For each retrieved tile, the amount of retained $\mathrm{D}$ in the surface was firstly analyzed by nuclear reaction analysis (NRA) using the $\mathrm{D}\left({ }^{3} \mathrm{He}, \mathrm{p}\right)^{4} \mathrm{He}$ reaction. In order to determine the quantitative $\mathrm{D}$ depth profile, the ${ }^{3} \mathrm{He}^{+}$incident energy was varied from 0.69 to $4.0 \mathrm{MeV}$, which allows measuring the $\mathrm{D}$ depth profile up to a depth of about $6 \mu \mathrm{m}$ [10]. The depth profiling measured points were mostly equivalent to the poloidal positions of divertor Langmuir probes. The morphology of tile surface was examined by scanning electron microscopy (SEM). After the surface analyses, the total amount of retained D, including the amount trapped in the bulk, was determined by thermal desorption spectroscopy (TDS). The tile was cut to smaller pieces, and each piece was heated from room temperature (RT) to $1270 \mathrm{~K}$ with a heating ramp of $\sim 0.25 \mathrm{~K} / \mathrm{s}$. Desorbed gases were analyzed by a quadrupole mass spectrometer. Comparison between NRA and TDS results can give the information about D diffusion and accumulation in the sample bulk.

\section{Result and Discussion}

\subsection{Characteristics of $D$ retention in different $W$ materials used in $A U G$}

Figure 2 shows D depth profiles obtained from 3 different measurement points on each $\mathrm{W}$ material. In general, the maximum D concentration is observed at the near surface region within several hundred $\mathrm{nm}$ in depth. Then, the concentration decreases with depth, and eventually, becomes roughly constant in the bulk $(>3 \mu \mathrm{m})$. One can find that D concentration in both VPS- and CMSII-W coatings is higher than that in solid bulk PC-W, e.g. VPS- and CMSII-W shows D concentrations of $\sim 0.1$ at.\% at depths of $>3 \mu \mathrm{m}$ whereas it is in the order of $10^{-3}$ at. $\%$ in the case of PC-W. 
$\mathrm{D}_{2}$ thermal desorption spectra from each $\mathrm{W}$ material are shown in Figure 3. In each case, the desorption starts at the temperatures of $450-500 \mathrm{~K}$, which reasonably agrees with the range of mean surface temperature during a discharge. The majority of retained $\mathrm{D}$ was released in the temperature range from $500 \mathrm{~K}-900 \mathrm{~K}$, obviously with not a single peak but several different desorption peaks. Additionally, there is a release peak appearing around 1050-1100 K, particularly, in W coatings. The shape of desorption spectrum varies depending on the $\mathrm{W}$ material and the poloidal location.

\subsection{Comparison of AUG results and laboratory results}

$\mathrm{D}$ retention in $\mathrm{W}$ materials depends strongly on the microstructure [11-13]. W coatings are usually expected to have a higher density of defects such as vacancies, voids and pores compared to PC-W, which may work as D trapping site. For instance, the mass density of VPS-W is usually $90-95 \%$ of theoretical one [14-17], and a high density of micrometer-scale pores in the coatings can be always confirmed by SEM. The D concentration with the order of $10^{-1}$ at. $\%$ observed in the AUG VPS-W agrees with the laboratory result gained by Alimov et al. for the same VPS-W coating [17]. The CMSII-W coating has less porosity compared to VPS-W, and is structured with oriented, nano-sized fine grains [8]. Nevertheless, laboratory experiments have indicated that CMSII-W includes also high density of D trapping sites [18,19]. The result from AUG CMSII-W, showing an order of magnitude higher D concentration than in the solid bulk PC-W, is generally consistent with those laboratory observations.

Although PC-W has much lower inherent defect density compared to $\mathrm{W}$ coatings, D retention at depths of several $\mu \mathrm{m}$ can be enhanced due to the formation of blisters [20]. The blistering is commonly observed on PC-W samples after D exposure at temperatures $<700 \mathrm{~K}$ in laboratory experiments, while no clear blistering was found on the AUG solid bulk PC-W tile. It is fair to mention that recent probe experiment in AUG did find blisters on the PC-W surface, with the type that grains are pushed out by D gas accumulation in intergranular cracks, after $6 \times 10^{25} \mathrm{~m}^{-2}$ of divertor plasma exposure [21]. This indicates blister can be formed under AUG plasma condition as well as in laboratory experiments. A major difference between the tile and the probe lies on the surface finishing process, i.e. the tile had a technically-machined surface while the surface of the probe sample was nicely mirror-finished to remove the distorted layer on the top of machined surface. A laboratory experiment performed by Nishijima et al. found that the blister formation can be significantly suppressed on the artificially scratched surface compared to the mirror-finished surface [22]. This suggests that blistering can be somehow suppressed on the machined surface as well.

The D desorption pattern gives information about trapping natures for the trapped D. D release behaviour from $\mathrm{W}$ has been discussed for the last decades, suggesting possible trapping natures and corresponding trapping energies as, e.g. (i) D trapped in weak traps as dislocations / grain boundaries with the effective trapping energy: $E_{\text {trap }}$ of $0.6-0.9 \mathrm{eV}$, (ii) the second $\mathrm{D}$ trapped in a vacancy / $\mathrm{D}_{2}-$ 
vacancy complex with $\mathrm{E}_{\text {trap }} \sim 1.0-1.1 \mathrm{eV}$, (iii) a $\mathrm{D}$ atom in a vacancy with $\mathrm{E}_{\text {trap }} \sim 1.2-1.3 \mathrm{eV}$, (iv) vacancy-clusters with $\mathrm{E}_{\text {trap }} \sim 1.4-1.5 \mathrm{eV}$ and $(\mathrm{v})$ chemisorption on the inner surface of a void $\left(\mathrm{E}_{\text {trap }} \sim\right.$ $1.8-2.1 \mathrm{eV}$ ) [23-28]. Since the AUG divertor tiles are normally heated up to $\sim 450 \mathrm{~K}$ or above, weak traps as (i), of which the D release peak is expected to appear at around $370-470 \mathrm{~K}$ in the present TDS condition, is not pronounced, but the contributions of traps (ii) - (v) are likely dominant. It should be kept in mind that there can be some other contribution such as C-D bond for high temperature desorption. Even after eliminating carbon plasma-facing surfaces, there is still non-negligible carbon impurity in the plasma and thereby a certain amount of carbon depositions. Although the outer divertor target is erosion-dominant region, there can be some depositions at local shadowed area due to the surface roughness and in the microscopic pores (such pores can be found, particularly, on the VPS-W coating).

Finally, D retention in AUG W materials as a function of incident fluence is summarized in Figure 4 together with relevant laboratory data [16,17,19,27,29-32] and an estimation of $\mathrm{D}$ in $\mathrm{W}$ compiled by the ITPA SOL/DIV topical group [33]. The thin CMSII-W coating shows that D retention determined by TDS (total retention) agrees relatively well to that measured by NRA (surface retention). This indicates D diffusion and accumulation in the graphite substrate is limited, and the retained D is mostly in the CMSII-W coating with the thickness of $10 \mu \mathrm{m}$ which is comparable to the NRA detectable range. On the other hand, the TDS results leaps upward from the NRA data for thicker VPS-W coating and solid $\mathrm{W}$ tile samples, indicating that more $\mathrm{D}$ is accumulated in the bulk beyond the NRA analysis depth. The retention level of the present bulk PC-W tile is lower compared to previous bulk PC-W probe data [7, 34], which is a positive aspect from the viewpoint of the tritium inventory issue, but the reason is not yet clear. The increase of D retention in the AUG solid bulk W determined by TDS shows nearly a square-root dependence from the incident fluence, again suggesting that the diffusion-limited trapping process plays a role in the retention. The ITPA SOL/DIV prediction shows a quasi-saturation of hydrogen inventory at fluences of $>10^{26} \mathrm{~m}^{-2}$ whereas the AUG data show no clear indication of the saturation so far. Nevertheless, the overall retention behaviour in AUG W materials is still not far from the predictions extrapolated from laboratory studies.

\section{Summary}

D retention in VPS- and CMSII-W coatings and solid bulk PC-W used as the outer divertor target of AUG in the last campaigns was investigated and compared with laboratory results. Since W coatings include high inherent defect density, the D concentration in both VPS- and CMSII-W coatings was higher than in solid bulk PC-W, which is consistent with laboratory data. No clear blistering was found on the solid bulk PC-W, which could be due to its technically-machined surface. The thin 
CMSII-W coating shows that D retention determined by TDS (total retention) agreed relatively well to that measured by NRA (surface retention) whereas TDS results showed much higher D retention compared to NRA results for thicker VPS-W coating and solid W tile samples, indicating that more D is accumulated in the bulk beyond the NRA detectable depth. The increase of D retention in the AUG solid bulk W shows nearly square-root dependence from the incident fluence. Those results indicate that diffusion-limited trapping process plays a key role for the retention. The overall retention behaviour in AUG W materials is fairly in the range of the predictions based on laboratory studies, which confirms the validity of the laboratory results under tokamak exposure conditions.

\section{Acknowledgments}

The authors appreciate J. Dorner, Th. Dürbeck and M. Fußeder for their technical assistance in the post-mortem measurement. 


\section{References}

[1] Philipps V 2011 J. Nucl. Mater. 415 S2

[2] Pitts R A 2009 Phys. Scr. T138 014001

[3] Roth J and Schmid K 2011 Phys. Scr. T145 014031, and references therein.

[4] Neu R et al. 2013 J. Nucl. Mater. 438 S34

[5] Mayer M et al. 2009 J. Nucl. Mater. 390-391 538

[6] Rohde V et al. 2009 Plasma Phys. Control. Fusion 51124033

[7] Sugiyama K et al.2010 Nucl. Fusion 50035001

[8] Ruset C et al. 2007 Phys. Scr. T128 171

[9] Herrmann A et al. 2011 Phys. Scr. T145 014068

[10] Mayer M et al. 2009 Nucl. Instrum. Meth. Phys. Res. B 267506

[11] Ogorodnikova O et al. 2009 Phys.Scr. 2009 T138 014053

[12] Manhard A et al. 2011 J. Nucl. Mater. 415 S632

[13] Balden M et al., J. Nucl. Mater. to be submitted

[14] García-Rosales C et al. 1996 J. Nucl. Mater. 233-237 803

[15] Tokunaga Ket al. 2002 J. Nucl. Mater. 307-311 126

[16] Golubeva A et al. "Hydrogen retention in plasma-sprayed tungsten" In Hydrogen in Matter: A Collection from the Papers Presented at the 2nd International Symposium on Hydrogen in Matter; ISOHIM (2006) vol. 837 of AIP Conference Proceedings

[17] Alimov V Kh et al. 2011 J. Nucl. Mater. 415 S628

[18] Zajec B et al. 2011 J. Nucl. Mater. 412116

[19] Ogorodnikova O et al. $2011 \mathrm{~J}$. Nucl. Mater. 419194

[20] Lindig S et al. 2011 Phys. Scr. T145 014039, and references therein.

[21] Balden M et al. 2013 J. Nucl. Mater. 438 S220

[22] Nishijima D et al., 2005 Nucl. Fusion 45669

[23] van Veen A et al. 1988 J. Nucl. Mater. 155-157 1113

[24] Fransens J R et al. 1991 J. Phys. Cond. Mat. 39871

[25] Eleveld H, van Veen A 1994 J. Nucl. Mater. 212-215 1421

[26] Alimov V Kh et al. 2001 Phys. Scr. T94 34

[27] Ogorodnikova O et al. 2003 J. Nucl. Mater. 313-316 469

[28] Poon M et al. 2008 J. Nucl. Mater. 374390

[29] Haasz A A et al. 1998 J. Nucl. Mater. 258-263 889

[30] Venhaus T et al. $2001 \mathrm{~J}$. Nucl. Mater. 290-293 505

[31] Roszell J P et al. 2011 J. Nucl. Mater. 415 S641

[32] Alimov V Kh et al. 2011 J. Nucl. Mater. 417572

[33] Lipschultz B et al. "An assessment of the current data affecting tritium retention and its use to project towards T retention in ITER" 2010 MIT Report PSFC/RR-10-4

[34] Sugiyama K et al. 2011 Phys. Scr. T145 014033 


\section{Figure captions}

Figure 1.

Distributions of outer divertor strike-point residence time and ion fluence (measured by divertor Langmuir probes) during the (a) 2007 (together with schematic view of the divertor), (b) 2009 and (c) 2010/11 campaigns of AUG. The poloidal scale in AUG divertor is given as "s-coordinate" starting from the upper edge of the inner divertor. The outer divertor target is in the range from $\mathrm{s}=1.027 \mathrm{~m}$ (lower edge) to $1.262 \mathrm{~m}$ as shown in (a) (inset).

Figure 2.

D depth profiles obtained from (a) VPS-W (2007), (b) CMSII-W (2009) and (c) solid bulk PC-W tiles. The profiles are from near the strike point (circle), high-fluence region (triangle) and the region far from strike point region (square) on each tile. For the s-coordinate, see figure 1.

Figure 3.

$\mathrm{D}_{2}$ desorption spectra obtained from (a) VPS-W (2007), (b) CMSII-W (2009) and (c) solid bulk PC-W tiles. Each spectrum was obtained from the sample cut out from the tile, and the poloidal location of each sample is given as s-coordinate. For the s-coordinate, see figure 1. Note that the scale for the desorption rate is different for each $\mathrm{W}$ material.

Figure 4.

Fluence dependences of D retention in (a) W coatings and (b) solid bulk PC-W: present AUG tile results together with the data previously obtained from AUG divertor probes [7, 34] and laboratory results $[16,17,19,27,30-32]$. The gray shaded region corresponds to an estimation of D retention in W based on laboratory experiments compiled by ITPA SOL/DIV topical group [33]. 


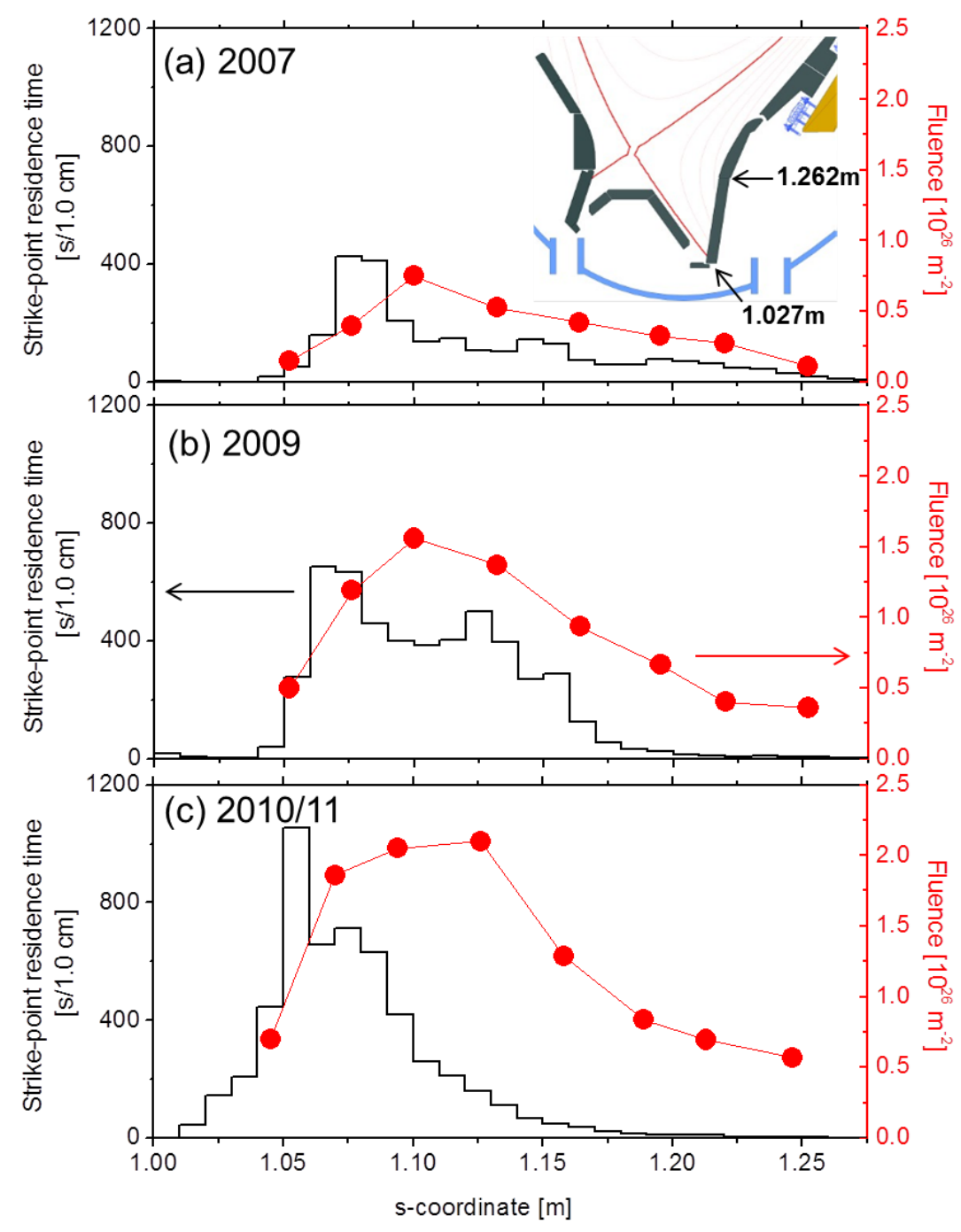

Figure 1

Distributions of outer divertor strike-point residence time and ion fluence (measured by divertor Langmuir probes) during the (a) 2007 (together with schematic view of the divertor), (b) 2009 and (c) 2010/11 campaigns of AUG. The poloidal scale in AUG divertor is given as "s-coordinate" starting from the upper edge of the inner divertor. The outer divertor target is in the range from $\mathrm{s}=1.027 \mathrm{~m}$ (lower edge) to $1.262 \mathrm{~m}$ as shown in (a) (inset). 


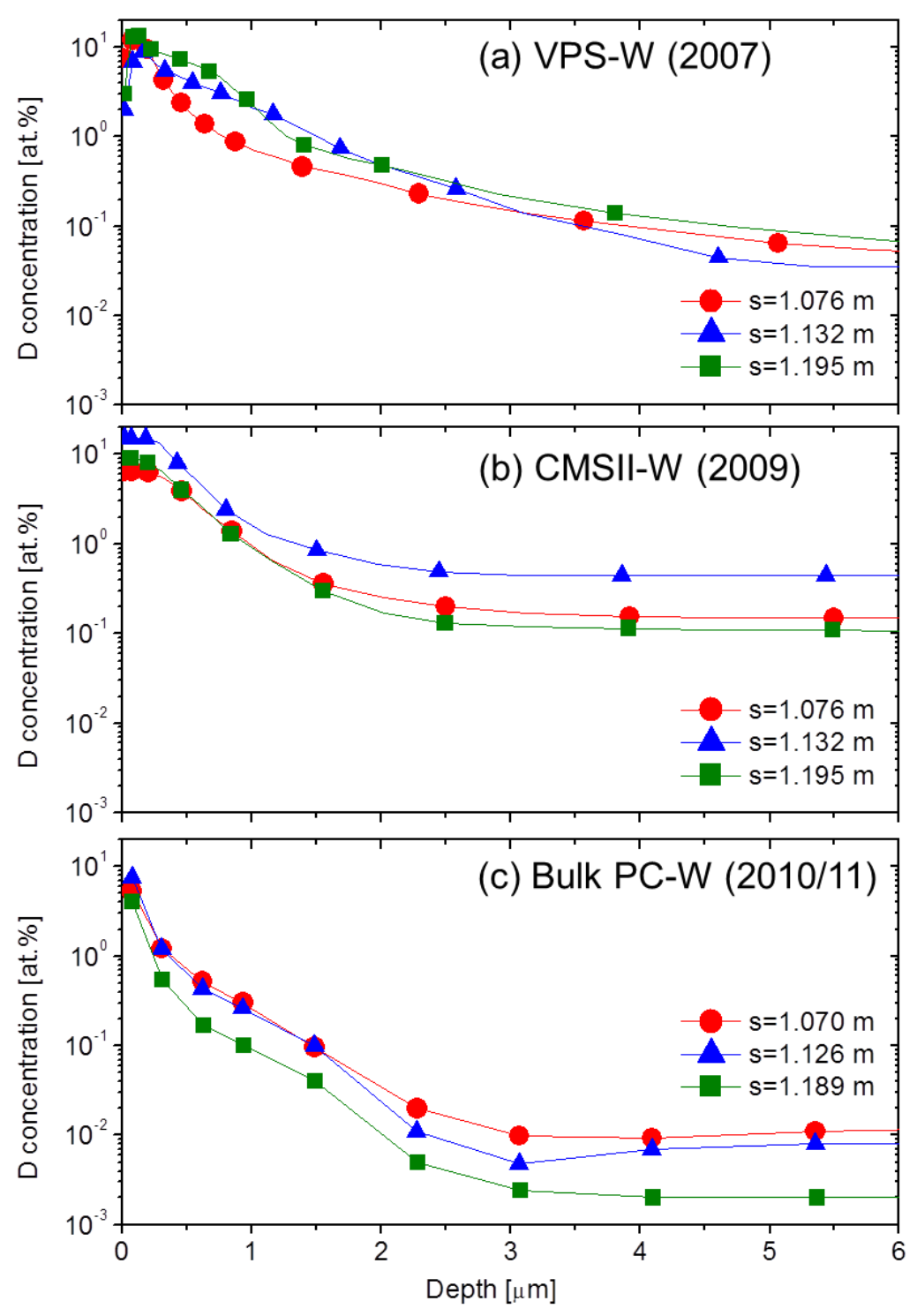

Figure 2

D depth profiles obtained from (a) VPS-W (2007), (b) CMSII-W (2009) and (c) solid bulk PC-W tiles. The profiles are from near the strike point (circle), high-fluence region (triangle) and the region far from strike point region (square) on each tile. For the s-coordinate, see figure 1. 


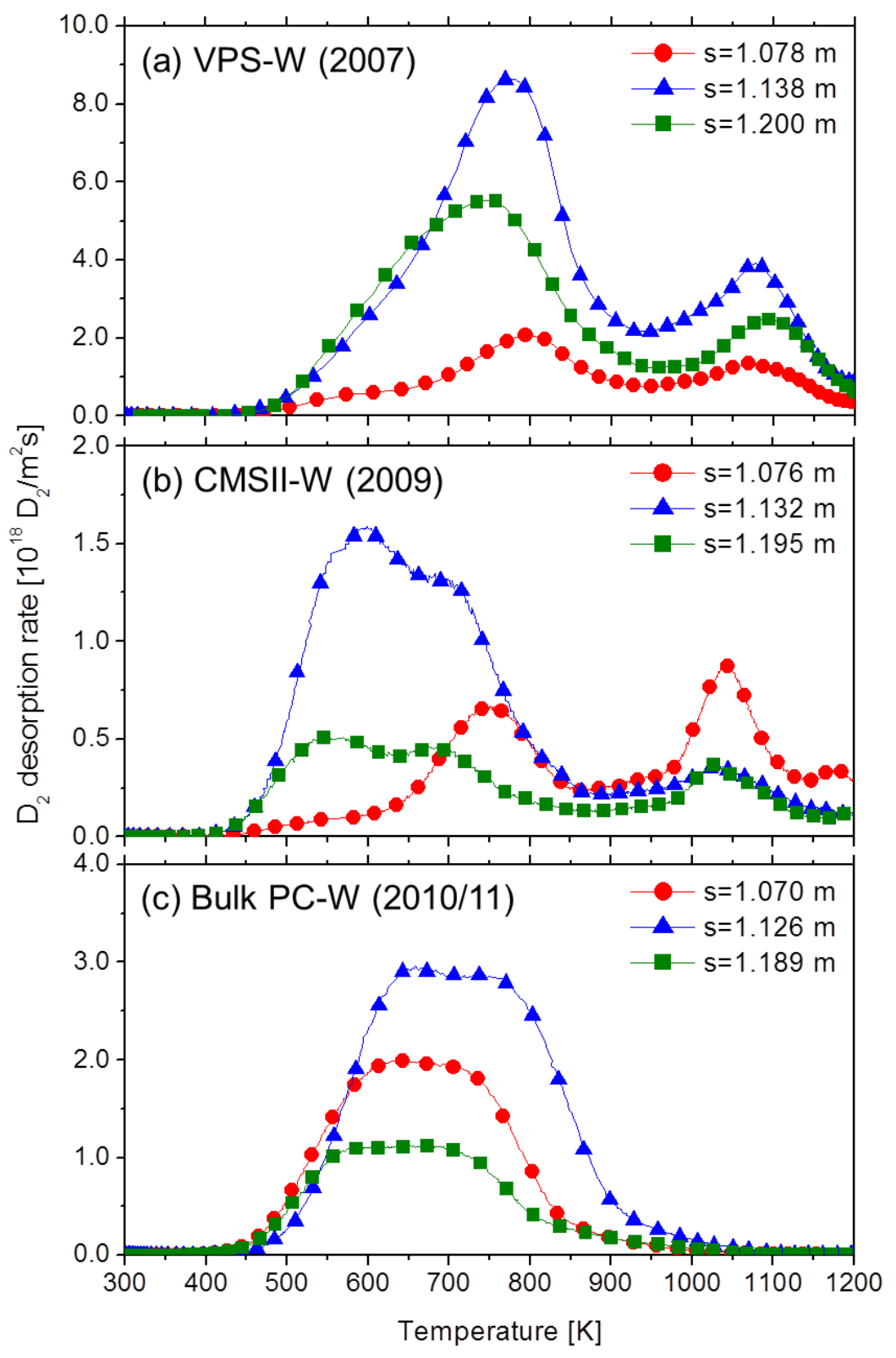

Figure 3

$\mathrm{D}_{2}$ desorption spectra obtained from (a) VPS-W (2007), (b) CMSII-W (2009) and (c) solid bulk PC-W tiles. Each spectrum was obtained from the sample cut out from the tile, and the poloidal location of each sample is given as s-coordinate. For the s-coordinate, see figure 1 . Note that the scale for the desorption rate is different for each $\mathrm{W}$ material. 

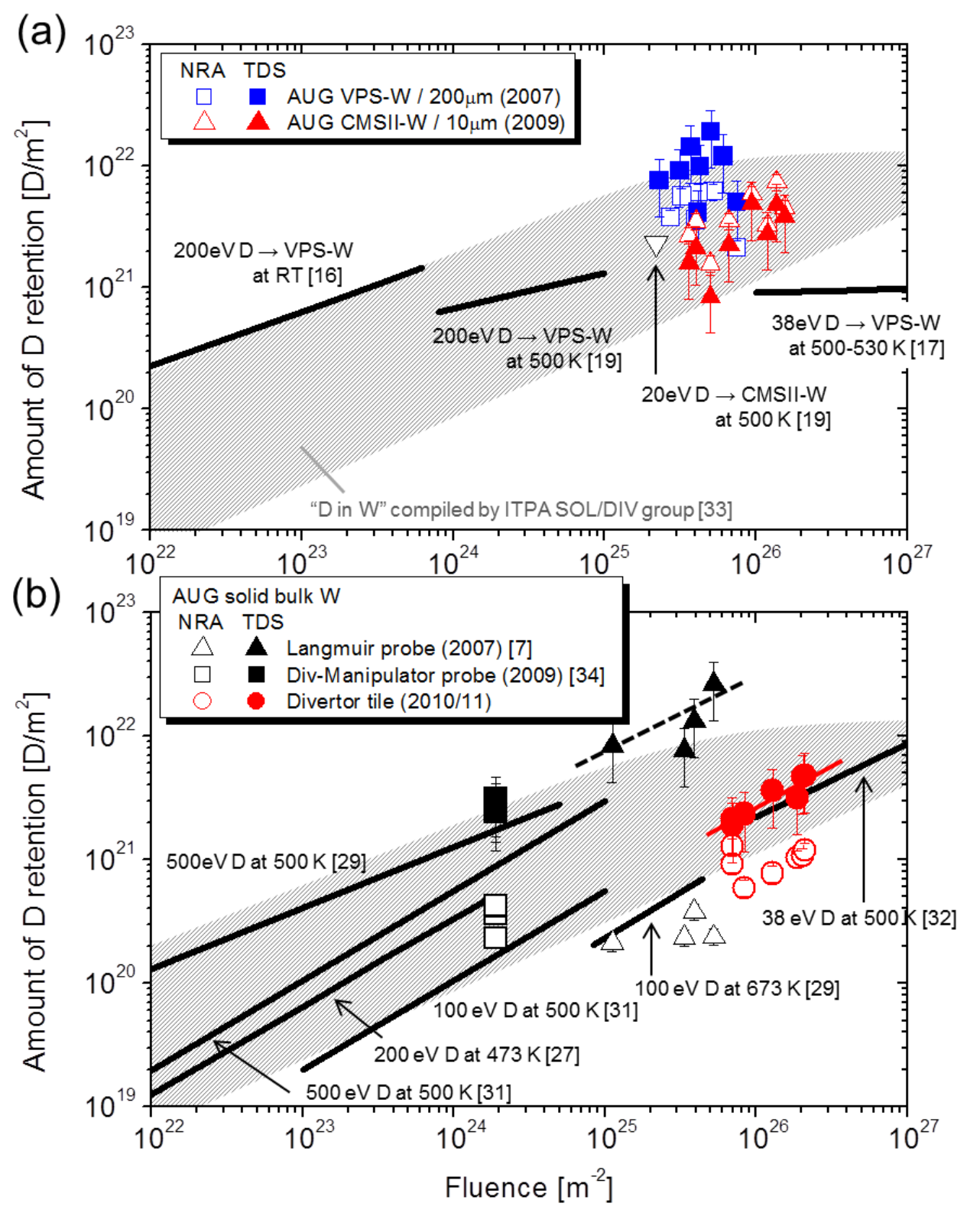

Figure 4

Fluence dependences of $\mathrm{D}$ retention in (a) $\mathrm{W}$ coatings and (b) solid bulk PC-W: present AUG tile results together with the data previously obtained from AUG divertor probes [7, 34] and laboratory results $[16,17,19,27,30-32]$. The gray shaded region corresponds to an estimation of $\mathrm{D}$ retention in $\mathrm{W}$ based on laboratory experiments compiled by ITPA SOL/DIV topical group [33]. 